OPEN ACCESS

Edited by:

Francisco Ciruela, University of Barcelona,

Spain

Reviewed by:

Stefano Comai,

Vita-Salute San Raffaele University,

Clement Zai,

Centre for Addiction and Mental Health (CAMH), Canada

*Correspondence: Alessio Squassina squassina@unica.it

Specialty section:

This article was submitted to

Neuropharmacology,

a section of the journal

Frontiers in Pharmacology

Received: 15 February 2019

Accepted: 15 May 2019

Published: 29 May 2019

Citation:

Pisanu C and Squassina A (2019)

Treatment Resistant Schizophrenia:

Insights from Genetic Studies and Machine Learning Approaches.

Front. Pharmacol. 10:617.

doi: 10.3389/fphar.2019.00617

\section{Treatment-Resistant Schizophrenia: Insights From Genetic Studies and Machine Learning Approaches}

\author{
Claudia Pisanu ${ }^{1,2}$ and Alessio Squassina ${ }^{1,3 *}$ \\ ${ }^{1}$ Department of Biomedical Sciences, Section of Neuroscience and Clinical Pharmacology, University of Cagliari, Cagliari, \\ Italy, ${ }^{2}$ Department of Neuroscience, Unit of Functional Pharmacology, Uppsala University, Uppsala, Sweden, ${ }^{3}$ Department \\ of Psychiatry, Dalhousie University, Halifax, NS, Canada
}

Schizophrenia (SCZ) is a severe psychiatric disorder affecting approximately 23 million people worldwide. It is considered the eighth leading cause of disability according to the World Health Organization and is associated with a significant reduction in life expectancy. Antipsychotics represent the first-choice treatment in SCZ, but approximately 30\% of patients fail to respond to acute treatment. These patients are generally defined as treatment-resistant and are eligible for clozapine treatment. Treatment-resistant patients show a more severe course of the disease, but it has been suggested that treatmentresistant schizophrenia (TRS) may constitute a distinct phenotype that is more than just a more severe form of SCZ. TRS is heritable, and genetics has been shown to play an important role in modulating response to antipsychotics. Important efforts have been put into place in order to better understand the genetic architecture of TRS, with the main goal of identifying reliable predictive markers that might improve the management and quality of life of TRS patients. However, the number of candidate gene and genome-wide association studies specifically focused on TRS is limited, and to date, findings do not allow the disentanglement of its polygenic nature. More recent studies implemented polygenic risk score, gene-based and machine learning methods to explore the genetics of TRS, reporting promising findings. In this review, we present an overview on the genetics of TRS, particularly focusing our discussion on studies implementing polygenic approaches.

Keywords: schizophrenia, antipsychotics, response, clozapine, pharmacogenetics, polygenic risk score

\section{INTRODUCTION}

Schizophrenia (SCZ) is a severe psychiatric disorder that affects approximately $1 \%$ of the general population and is associated with a significant socioeconomic burden (Kahn et al., 2015). Antipsychotics represent the mainstay treatment for SCZ, but around one-third of patients show no response (Gillespie et al., 2017). According to the American Psychiatric Association (APA) guidelines, treatment-resistant schizophrenia (TRS) patients are defined as those showing little or no response to at least two non-clozapine antipsychotic trials of adequate duration and dose range (Lehman et al., 2004). Clozapine is the only treatment with an indication for TRS. However, this drug is still underutilized due to monitoring requirement (Kelly et al., 2018) and potential adverse effects, some of which can be severe and life-threatening (Wheeler et al., 2009; Gillespie et al., 2017). Unfavorable response to first-line pharmacological treatments is generally associated with a more 
severe course of disease in TRS patients (Gillespie et al., 2017; Nucifora et al., 2018). Moreover, TRS patients are highly exposed to the potential detrimental effect of inefficacious treatments, including risk for adverse reactions that could be obviated or reduced if treatment resistance was known in advance.

It has been suggested that differential treatment response to antipsychotics might underlie biologically distinct subphenotypes of SCZ (Farooq et al., 2013; Gillespie et al., 2017) and TRS might better constitute a distinct phenotype rather than just a more severe form of SCZ (Wimberley et al., 2017). In this scenario, it has become clear that TRS patients would significantly benefit from the identification of clinical and biological markers to possibly predict the risk for treatment resistance before starting pharmacological treatments. However, TRS is poorly understood and its neurobiological underpinnings have yet to be clarified.

Data from family studies suggest that TRS is a heritable trait and that heritability might be stronger than in responsive SCZ (Nucifora et al., 2018). Candidate gene and genome-wide association studies (GWAS) have investigated how genetic variation might explain the interindividual variability observed in response to antipsychotics, but only a limited number of them focused on TRS (Nucifora et al., 2018). The majority of these studies used clozapine prescription or treatment as a proxy for diagnosis of TRS, and the investigated genetic variants were selected mainly based on their previously association with SCZ. We can anticipate that findings from these studies have so far not allowed dissecting the genetic complexity underlining TRS. Indeed, it has become clear that TRS is characterized by a complex polygenic nature. Most recent investigations have applied novel approaches such as polygenic risk score (PRS) and kernel support vector machine (SVM) to aggregate the effects of multiple variants contributing to disease risk. These approaches might be better able to capture the polygenic architecture of psychiatric conditions as well as response to psychotropic medications. In light of these observations, in this article, we will review studies investigating the genetic bases of TRS with a focus on studies using polygenic analytical approaches. The articles described in this narrative review were retrieved through a search on PubMed using the following keywords: treatment-resistant schizophrenia, antipsychotic response, genome-wide association study, machine learning, polygenic risk score, and support vector machine.

\section{OVERLAP BETWEEN PREDISPOSITION TO SCZ AND RESPONSE TO ANTIPSYCHOTICS}

As in the case of other psychiatric disorders, pharmacogenetic studies of antipsychotics have explored the overlap between susceptibility to SCZ and probability to respond to pharmacological treatments. GWASs successfully identified a large number of underlying genetic loci involved in SCZ. Among the most significant efforts, a first mega-analysis of GWAS conducted by the Psychiatric Genomics Consortium (PGC), including 9,394 cases with SCZ and 12,462 controls of European origin, had identified seven genome-wide significant loci (Schizophrenia Psychiatric Genome-Wide Association Study (GWAS) Consortium, 2011). These results were replicated by a GWAS in which patients with
SCZ treated with clozapine were compared with healthy controls (Hamshere et al., 2013). More recently, the second mega-analysis of GWAS conducted by the PGC (wave 2), including 36,989 cases and 113,075 controls, identified 108 loci associated with predisposition to SCZ (Schizophrenia Working Group of the Psychiatric Genomics Consortium, 2014). Besides single-nucleotide polymorphisms (SNPs), rare disruptive mutations identified with exome sequencing have also been shown to increase liability for SCZ (Purcell et al., 2014). Based on the evidence that many of the variants identified by genome-wide studies on SCZ are located in genes playing a role in systems likely involved in its neurobiology, there is a rationale for investigating the association between genetic risk for $\mathrm{SCZ}$ and response to antipsychotics or TRS. Ruderfer et al. (2016) evaluated both common and rare SCZ-associated loci for enrichment in drug targets, providing interesting evidence that supports the role of some of these genes as drug targets. Authors used a geneset analysis approach and found that $21 \%$ of 167 pharmacological subgroups were enriched for loci previously associated with SCZ. Dopamine receptor D2 (DRD2) was among the loci contributing the most to this finding. Indeed, $D R D 2$ encodes a known target not only of antipsychotics, but also of 46 different non-antipsychotic pharmacological subgroups out of the 167 evaluated in Ruderfer et al. (2016). The gene set including targets of antipsychotics was enriched for common and rare variants previously associated with SCZ. Authors also compared TRS (532 SCZ patients treated with clozapine) with SCZ patients treated with other antipsychotics $(n=2,002)$, showing a higher number of disruptive mutations in genes targeted by antipsychotics in the TRS group. Taken together, these results support the hypothesis that at least some of the genes identified as involved in the pathogenesis of SCZ might also explain part of the interindividual variability in response to antipsychotics as well as in susceptibility to TRS.

\section{GENETIC BASES OF TRS}

The number of studies exploring the correlation between genetic variants and TRS is relatively limited. There is still disagreement on the best approach to maximize the power and informativity of genetic studies on TRS, considering the likely complex genetic architecture of this phenotype and the discrepancies on the clinical definition of TRS. The most used operational criteria to define TRS were provided by Kane and colleagues in 1988 (Kane et al., 1988). These criteria include 1) three or more periods of treatment with at least two neuroleptic agents of different classes (1,000 mg/day of chlorpromazine equivalents) for at least 6 weeks, 2) no period of good functioning within the preceding 5 years, and 3) severe psychopathology according to the Brief Psychiatric Rating Scale (BPRS) and Clinical Global Impressions (CGI) scores (Kane et al., 1988). These criteria include more aspects related to functioning compared to the APA guidelines that, as mentioned in the Introduction section, define TRS as little or no response to at least two non-clozapine antipsychotic trials of adequate duration and dose range (Lehman et al., 2004).

Several studies that will be presented in this section used the APA guidelines or a modified version of the criteria defined by Kane et al. (1988), while others used clozapine use or prescription 
and discontinuation from the prescribed antipsychotic to select TRS patients. It is therefore consequential that the comparison of findings from studies using different definitions may not be straightforward. However, taken together, findings in this field further highlight the importance of the genetic contribution in characterizing TRS.

\section{Candidate Gene and GWAS}

Candidate gene studies investigating the involvement of specific targets in TRS mostly focused on the dopaminergic and serotoninergic systems (Inada et al., 2003; Ji et al., 2008; Kohlrausch et al., 2008; Ota et al., 2012; Bilic et al., 2014; Terzić et al., 2015), as well as on systems involved in inflammation and oxidative stress (Jia et al., 2011; Pinheiro et al., 2017). Among the most interesting findings, Bilic et al. (2014) reported significant interactions between a dopamine transporter variable number tandem repeat (DATVNTR) and the serotonin transporter (SERT)-in2 polymorphism in a sample of 172 patients with SCZ, 92 of whom met the TRS definition based on the modified Kane criteria: 1) at least 5 years of inadequate social or occupational functioning, 2) current treatment with a chlorpromazine equivalent dose $>600 \mathrm{mg}$ or score $\geq 3$ on selected items of the Positive and Negative Schizophrenic Symptoms (PANSS) scale or Clinical Global Impression-Severity scale (CGIS) score $\geq 4$, and 3) history of previous treatment with at least two antipsychotics or history of at least one treatment with clozapine. Results from this study support the hypothesis that, besides the main effect of specific genetic variants, SNP-SNP interactions might also play a role in explaining the interindividual variance observed in response to antipsychotics.

The first large-scale study on the genetic bases underlying response to antipsychotics was conducted in a sample recruited within the Clinical Antipsychotic Trials of Intervention Effectiveness (CATIE) trial (Need et al., 2009). Although this study (which tested 769 polymorphisms in 118 candidate genes) reported several nominal associations, no variant was significantly associated with response to antipsychotics after correction for multiple testing (Need et al., 2009). This study did not specifically include patients with TRS and used discontinuation from the prescribed antipsychotic as a proxy for non-response.

As for large-scale studies, only a small number were conducted in TRS patients. Zhang and coworkers (2013) selected SNPs nominally associated with non-response in the CATIE pharmacogenetic study (Need et al., 2009) and tested their association with TRS (using clozapine treatment as a proxy). Authors reported a significant association between TRS and several genetic variants in linkage disequilibrium (top hit: rs11030104) located in the brain-derived neurotrophic factor (BDNF) gene. Li and Meltzer (2014) conducted a GWAS on two cohorts of Caucasian patients with ( $n=79$ and $n=$ 70 ) and without ( $n=95$ and $n=125)$ TRS. In this study, treatment resistance was defined as persistence of moderate to severe positive symptoms despite at least two trials of 4-6 weeks with typical or atypical antipsychotics other than clozapine. Although no SNP met the genome-wide significant threshold, interesting results were reported for the rs 2237457 variant located in $7 \mathrm{p} 12$ ( $p$ value in the combined cohorts: $\left.5.66 \times 10^{-7}\right)$. This variant is located upstream of the gene encoding L-dopa decarboxylase, a rate-limiting enzyme in the synthesis of trace amines and neurotransmitters, including dopamine ( $\mathrm{Li}$ and Meltzer, 2014). This result is particularly important based on the fact that a hyperdopaminergic state in the mesolimbic dopamine pathway is thought to play a crucial role in the development of psychotic symptoms according to the dopamine hypothesis of SCZ and that dopamine D2 receptors represent a main target for all antipsychotic drugs (Li et al., 2016).

Conversely, Teo and coworkers (2012) did not identify any significant association among 384 candidate gene loci in a study including 85 patients with TRS defined according to APA criteria and 155 non-resistant patients. Other studies focused on the association of genetic variants and clinical factors hypothesized to play a role in the development of TRS, such as childhood adversities. In a GWAS on a sample of 85 Caucasian patients with SCZ [31 of whom met the criteria for TRS defined according to the APA criteria (Lehman et al., 2004)], no SNP met the genomewide significant threshold for association with TRS with or without taking into account history of childhood adversities (Koga et al., 2017).

\section{Studies Using PRS}

PRS analysis aggregates the effect sizes of several SNPs across the genome, thus providing a single estimate of the association with a specific trait or disease (Dudbridge, 2013). In the last few years, PRS analysis has been successfully applied to the study of different psychiatric disorders (Bipolar Disorder and Schizophrenia Working Group of the Psychiatric Genomics Consortium, 2018; Kalman et al., 2018; Taylor et al., 2018; Richards et al., 2019). In the case of SCZ, PRS analysis has been used to evaluate how the polygenic burden can explain differences in specific symptoms (Wang et al., 2018; Anderson-Schmidt et al., 2019), functional and structural brain changes (Lieslehto et al., 2018; Ranlund et al., 2018; Velthorst et al., 2018), genetic overlap with other traits (International Consortium on Lithium Genetics (ConLi+Gen) et al., 2018), as well as gene co-expression networks in the brain (Radulescu et al., 2018). Studies investigating the potential value of PRS analysis to identify patients that are less likely to respond to treatment provided contrasting findings. In patients with first-episode psychosis (FEP), Zhang and colleagues (2018) found that participants with lower SCZ polygenic burden were more likely to respond to a 12-week antipsychotic treatment compared to patients with high SCZ PRS (odds ratio = 1.91). Conversely, although Santoro et al. (2018) reported a positive association between depressive symptoms and PRS at baseline in a sample of 60 FEP patients, after treatment with risperidone, patients with a higher SCZ PRS were more likely to show improvement in depressive and excitement symptoms. Of note, the study by Santoro and coworkers only included antipsychotic-naive patients.

Studies including patients with TRS also provided controversial findings (Table 1). Frank and coworkers (2015) reported that a PRS including SNPs associated with SCZ risk in the first meta-analysis from the PGC Schizophrenia group (Schizophrenia Psychiatric Genome-Wide Association Study (GWAS) Consortium, 2011) was increased in patients with SCZ treated with clozapine compared to patients not treated with this drug. Moreover, the highest PRS was observed in patients who are non-responders to clozapine 
TABLE 1 | Studies investigating the genetic bases of treatment-resistant schizophrenia (TRS) using a polygenic risk score (PRS)

\begin{tabular}{|c|c|c|c|c|}
\hline Study & Discovery sample & Target sample & $\begin{array}{c}\text { Treatment-resistance } \\
\text { criteria }\end{array}$ & Results \\
\hline Frank et al. (2015) & $\begin{array}{l}\text { First meta-analysis from the } \\
\text { PGC Schizophrenia group } \\
\text { (Schizophrenia Psychiatric } \\
\text { Genome-Wide Association Study } \\
\text { Consortium, 2011) }\end{array}$ & $\begin{array}{l}804 \text { German patients with } \\
\text { SCZ (434 with TRS) }\end{array}$ & History of clozapine treatment & $\begin{array}{l}\text { Higher PRS in patients treated with } \\
\text { clozapine compared to patients with } \\
\text { no history of clozapine treatment. The } \\
\text { highest PRS was observed in patients } \\
\text { characterized by non-response to } \\
\text { clozapine, early age at onset and poor } \\
\text { premorbid social functioning }\end{array}$ \\
\hline $\begin{array}{l}\text { Martin and Mowry } \\
\text { (2016) }\end{array}$ & $\begin{array}{l}\text { Second meta-analysis from } \\
\text { the PGC Schizophrenia group } \\
\text { (Schizophrenia Working Group } \\
\text { of the Psychiatric Genomics } \\
\text { Consortium, 2014) }\end{array}$ & $\begin{array}{l}612 \text { Australian patients } \\
\text { with SCZ (227 with TRS) }\end{array}$ & $\begin{array}{l}\text { Poor functioning, continuous } \\
\text { course of illness, and at } \\
\text { least two among delusions, } \\
\text { hallucinations, disorganization } \\
\text { and negative symptoms } \\
\text { during treatment with } \\
\text { antipsychotics }\end{array}$ & $\begin{array}{l}\text { No association between the PRS } \\
\text { and non-response to antipsychotics. } \\
\text { Association between TRS and total } \\
\text { duplication burden genome-wide }\end{array}$ \\
\hline Wimberley et al. (2017) & $\begin{array}{l}\text { Second meta-analysis from } \\
\text { the PGC Schizophrenia group } \\
\text { (Schizophrenia Working Group } \\
\text { of the Psychiatric Genomics } \\
\text { Consortium, 2014), excluding the } \\
\text { Danish cases }\end{array}$ & $\begin{array}{l}862 \text { participants with SCZ } \\
\text { (181 with TRS) included } \\
\text { in the Danish Newborn } \\
\text { Screening Biobank }\end{array}$ & $\begin{array}{l}\text { Clozapine initiation or } \\
\text { hospitalization during } \\
\text { antipsychotic treatment } \\
\text { after at least two periods } \\
\text { of different antipsychotics } \\
\text { monotherapy }\end{array}$ & $\begin{array}{l}\text { No association between the PRS } \\
\text { and TRS }\end{array}$ \\
\hline
\end{tabular}

PGC, psychiatric genomics consortium; PRS, polygenic risk score; SCZ, schizophrenia; TRS, treatment-resistant schizophrenia.

and characterized by early age at onset and poor premorbid social functioning (Frank et al., 2015).

On the other hand, using the population-based Danish register, Wimberley and coworkers found no association between a PRS for SCZ and TRS (Wimberley et al., 2017). In this study, the PRS was computed based on 24,755 SNPs from the PGC wave 2 paper, using a $p$-value threshold of 0.05 (Schizophrenia Working Group of the Psychiatric Genomics Consortium, 2014), and tested into an independent Danish cohort of 862 patients (181 of whom were considered to be treatment-resistant). In this study, treatment resistance was defined as either 1) clozapine initiation or 2) hospitalization during antipsychotic treatment after at least two periods of different antipsychotics monotherapy (Wimberley et al., 2017). Similarly, Martin and Mowry (2016) found no association between a PRS for SCZ and non-response to antipsychotics in a sample of 612 Australian patients with SCZ (227 of whom showed treatment resistance). In this study, patients were considered to be treatment-resistant in case they showed poor functioning, continuous course of illness, and at least two among delusions, hallucinations, disorganization, and negative symptoms during treatment with antipsychotics. Similarly to other works, the PRS was computed based on SNPs identified by the most recent PGC wave 2 GWAS mega-analysis (Schizophrenia Working Group of the Psychiatric Genomics Consortium, 2014), although a different $p$-value threshold was used $(p<0.1)$. Of note, the authors found a significant association between TRS and the total copy number duplication burden genome-wide (Martin and Mowry, 2016). A number of factors might explain the observed discrepancies between studies investigating genetic bases of TRS using PRS models. For instance, the different $p$-value thresholds chosen by the authors contribute to make these results difficult to compare. Moreover, the sample size of the available studies was generally limited, as the number of subjects with a diagnosis of TRS ranged from 181 to 434 (Table 1). To date, available studies only used PRS including SCZ risk variants. However, the development of a PRS specific for TRS might be of high relevance to better capture the contribution of loci underlying non-response to antipsychotics. Moreover, studies evaluating the contribution of a PRS together with clinical characteristics previously identified to be associated with TRS (e.g., earlier age at onset, family history of psychosis and history of substance abuse) would be of high interest.

\section{COMPARISON BETWEEN PRS AND OTHER MACHINE LEARNING METHODS}

PRS analysis aggregates the contribution of multiple SNPs assuming an additive effect. While this approach has proven to be extremely useful, it doesn't allow to take into account the potential interactions between different genetic variants. A recent study tried to address this gap using SVM algorithms (Vivian-Griffiths et al., 2019). SVM is a family of supervised learning methods that can be usefully applied to linear as well as non-linear and highdimensional classification problems (Cai et al., 2001). Specifically, the SVM method allows one to find the optimum hyperplane that separates observations into different classes. Vivian-Griffiths and coworkers (2019) evaluated how this method (allowing to take into account pairwise and higher-order SNP interactions) might help to distinguish patients with TRS from healthy controls compared to a PRS. Models were based on 1) 125 genome-wide significant SNPs and 2) 4,998 independent top SNPs from the PGC wave 2 GWAS mega-analysis (Schizophrenia Working Group of the Psychiatric Genomics Consortium, 2014). The study was conducted in the CLOZUK sample (Hamshere et al., 2013), including 5,554 patients receiving clozapine and 6,299 healthy controls. In this study, two different typologies of SVM models were constructed: 
SVM with linear and radial basis function kernels. Predictive performances were measured using the area under the receiver operating characteristics curve metric (AUC-ROC) and compared between these two models as well as against those of a PRS. The standardized reference allele counts for each polymorphism were used as input for the SVM model. While no evidence of interaction was found when analyzing the 125 top hits, findings from this study suggested the potential presence of interactions in the models including the more weakly SCZ-associated variants (Vivian-Griffiths et al., 2019). Although the SVM method might be more suitable to take into account these effects, the PRS model showed a higher accuracy in the classification of patients with SCZ treated with clozapine compared with controls. Nonetheless, the prediction accuracy shown by the PRS is still insufficient to support the implementation of the model into the clinical practice [best area under the receiver operating characteristics curve $($ AUC-ROC $)=0.697]$.

\section{CONCLUSIONS}

A growing number of studies provided intriguing hints on the complex polygenic architecture underlying TRS. However, to date, no genetic marker showed adequate prediction accuracy. Moreover, controversial findings were reported as regards the ability of available PRSs to discriminate individuals with or without TRS. The majority of available studies investigated the effect of variants previously associated with predisposition to SCZ. Indeed, it has been shown that antipsychotics' targets are enriched for variants previously associated with SCZ (Ruderfer et al., 2016), supporting the need of investigating a potential role for these genes in TRS development. However, in order to better capture the contribution of variants specifically implicated in response to antipsychotics, a more comprehensive approach might involve construction of a PRS specific for TRS (i.e., constructed using genetic data from patients characterized for response to antipsychotics) as such a score might show a higher predictive accuracy. The importance of this aspect is highlighted by the fact that the total duplication burden genome-wide has been associated with TRS (Martin and Mowry, 2016) but not with predisposition to SCZ (Buizer-Voskamp and Muntjewerff, 2011), supporting the hypothesis that only a part of TRS susceptibility might be explained by previously investigated targets.

\section{REFERENCES}

Anderson-Schmidt, H., Gade, K., Malzahn, D., Papiol, S., Budde, M., Heilbronner, U., et al. (2019). The influence of religious activity and polygenic schizophrenia risk on religious delusions in schizophrenia. Schizophr. Res. pii: S0920-9964(18)307217. doi: 10.1016/j.schres.2018.12.025

Bilic, P., Jukic, V., Vilibic, M., Savic, A., and Bozina, N. (2014). Treatment-resistant schizophrenia and DAT and SERT polymorphisms. Gene 543 (1), 125-132. doi: 10.1016/j.gene.2014.03.050

Bipolar Disorder and Schizophrenia Working Group of the Psychiatric Genomics Consortium. (2018). Genomic dissection of bipolar disorder and schizophrenia, including 28 subphenotypes. Cell 173, 1705-1715.e16. doi: 10.1016/j.cell.2018. 05.046
Another aspect that limits our interpretation of available studies consists in the different criteria used by different researchers to define TRS. While some of the studies adopted the criteria suggested by APA (which define TRS as little or no response to at least two non-clozapine antipsychotic trials of adequate duration and dose range), other studies used different criteria [e.g., modified versions of the Kane criteria (Kane et al., 1988), which take into account different aspects, including global assessment of functioning] or simply considered history of clozapine treatment as a proxy of TRS. Although the latter approach is easier to apply (particularly in the case of studies performed using data from registers) and can therefore lead to studies with an increased sample size, it might lead to an underestimation of the number of patients with TRS. In fact, although clozapine is the only antipsychotic with an indication for TRS, this drug is currently underutilized due to possibly life-threatening adverse reactions as well as to the need of regular monitoring (Remington et al., 2016). In order to minimize this risk, some studies included not only patients treated with clozapine but also participants who would meet the criteria for initiating clozapine treatment based on the data extracted from population-based registers (Wimberley et al., 2017). Nevertheless, the use of standardized criteria to identify patients affected by TRS should be one of the main goals of future efforts, as this would allow evaluating the reproducibility and robustness of findings and the aggregation of available results in meta-analyses, ultimately leading us closer to the understanding of the genetic architecture of TRS.

\section{AUTHOR CONTRIBUTIONS}

AS and CP searched the literature and compiled the review with equal contribution.

\section{FUNDING}

CP is supported by Fondazione di Sardegna ("Convenzione triennale 2015-2017") and by the Autonomous Region of Sardinia (“Legge Regionale 7 agosto 2007, n. 7 - call 2016"), grant number F72F16003090002. Publication fees were covered with funding from a project funded by Regione Atonoma della Sardegna, L.R. 7, call 2013, grant number F72I15000830002.

Buizer-Voskamp, J. E., and Muntjewerff, J. W. (2011). Genetic Risk and Outcome in Psychosis (GROUP) Consortium Members, Strengman E, Sabatti C, Stefansson H, Vorstman JA, Ophoff RA. Genome-wide analysis shows increased frequency of copy number variation deletions in Dutch schizophrenia patients. Biol. Psychiatry 70 (7), 655-662. doi: 10.1016/j.biopsych.2011.02.015

Cai, Y. D., Liu, X. J., Xu, X., and Zhou, G. P. (2001). Support vector machines for predicting protein structural class. BMC Bioinformatics 2, 3. doi: 10.1186/ 1471-2105-2-3

Dudbridge, F. (2013). Power and predictive accuracy of polygenic risk scores. PLoS Genet. 9 (3), e1003348. doi: 10.1371/annotation/b91ba224-10be-409d-93f4 $-7423 \mathrm{~d} 502 \mathrm{cba} 0$

Farooq, S., Agid, O., Foussias, G., and Remington, G. (2013). Using treatment response to subtype schizophrenia: proposal for a new paradigm in classification. Schizophr. Bull. 39 (6), 1169-1172. doi: 10.1093/schbul/sbt137 
Frank, J., Lang, M., Witt, S. H., Strohmaier, J., Rujescu, D., Cichon, S., et al. (2015). Identification of increased genetic risk scores for schizophrenia in treatmentresistant patients. Mol. Psychiatry 20 (7), 913. doi: 10.1038/mp.2015.52

Gillespie, A. L., Samanaite, R., Mill, J., Egerton, A., and MacCabe, J. H. (2017). Is treatment-resistant schizophrenia categorically distinct from treatmentresponsive schizophrenia? A systematic review. BMC Psychiatry 17 (1), 12. doi: 10.1186/s12888-016-1177-y

Hamshere, M. L., Walters, J. T., Smith, R., Richards, A. L., Green, E., Grozeva, D., et al. (2013). Genome-wide significant associations in schizophrenia to ITIH3/4, CACNA1C and SDCCAG8, and extensive replication of associations reported by the Schizophrenia PGC. Mol. Psychiatry 18 (6), 708-712. doi: 10.1038/mp.2012.67

Inada, T., Nakamura, A., and Iijima, Y. (2003). Relationship between catechol-Omethyltransferase polymorphism and treatment-resistant schizophrenia. Am. J. Med. Genet. B Neuropsychiatr. Genet. 120B (1), 35-39. doi: 10.1002/ajmg. b. 20023

International Consortium on Lithium Genetics (ConLi+Gen), Amare, A. T., Schubert, K. O., Hou, L., Clark, S. R., Papiol, S., et al. (2018). Association of polygenic score for schizophrenia and HLA antigen and inflammation genes with response to lithium in bipolar affective disorder: a genome-wide association study. JAMA Psychiatry 75 (1), 65-74. doi: 10.1001/jamapsychiatry.2017.3433

Ji, X., Takahashi, N., Saito, S., Ishihara, R., Maeno, N., Inada, T., et al. (2008). Relationship between three serotonin receptor subtypes (HTR3A, HTR2A and HTR4) and treatment-resistant schizophrenia in the Japanese population. Neurosci. Lett. 435 (2), 95-98. doi: 10.1016/j.neulet.2008.01.083

Jia, P., Jayathilake, K., Zhao, Z., and Meltzer, H. Y. (2011). Association of FAS, a TNF- $\alpha$ receptor gene, with treatment resistant schizophrenia. Schizophr Res. 129 (2-3), 211-212. doi: 10.1016/j.schres.2011.04.013

Kahn, R. S., Sommer, I. E., Murray, R. M., Meyer-Lindenberg, A., Weinberger, D. R., Cannon, T. D., et al. (2015). Schizophrenia. Nat. Rev. Dis. Primers. 1, 15067. doi: $10.1038 /$ nrdp. 2015.67

Kalman, J., Papiol, S., Forstner, A. J., Heilbronner, U., Degenhardt, F., Strohmaier, J., et al. (2018). Investigating polygenic burden in age at disease onset in bipolar disorder: findings from an international multicentric study. Bipolar Disord. 21 (1), 68-75. doi: 10.1111/bdi.12659

Kane, J., Honigfeld, G., Singer, J., and Meltzer, H. (1988). Clozapine for the treatmentresistant schizophrenic. A double-blind comparison with chlorpromazine. Arch Gen. Psychiatry 45 (9), 789-796. doi: 10.1001/archpsyc.1988.01800330013001

Kelly, D. L., Freudenreich, O., Sayer, M. A., and Love, R. C. (2018). Addressing barriers to clozapine underutilization: a national effort. Psychiatr. Serv. 69 (2), 224-227. doi: 10.1176/appi.ps.201700162

Koga, A., Bani-Fatemi, A., Hettige, N., Borlido, C., Zai, C., Strauss, J., et al. (2017). GWAS analysis of treatment resistant schizophrenia: interaction effect of childhood trauma. Pharmacogenomics 18 (7), 663-671. doi: 10.2217/pgs-2016-0137

Kohlrausch, F. B., Gama, C. S., Lobato, M. I., Belmonte-de-Abreu, P., CallegariJacques, S. M., Gesteira, A., et al. (2008). Naturalistic pharmacogenetic study of treatment resistance to typical neuroleptics in European-Brazilian schizophrenics. Pharmacogenet. Genomics. 18 (7), 599-609. doi: 10.1097/FPC.0b013e328301a763

Lehman, A. F., Lieberman, J. A., Dixon, L. B., McGlashan, T. H., Miller, A. L., Perkins, D. O., et al. (2004). Practice guideline for the treatment of patients with schizophrenia, 2nd edition. Am. J. Psychiatry 161 (2 Suppl), 1-56.

Li, P., Snyder, G. L., and Vanover, K. E. (2016). Dopamine targeting drugs for the treatment of schizophrenia: past, present and future. Curr. Top Med. Chem. 16 (29), 3385-3403. doi: 10.2174/1568026616666160608084834

Li, J., and Meltzer, H. Y. (2014). A genetic locus in 7p12.2 associated with treatment resistant schizophrenia. Schizophr Res. 159 (2-3), 333-339. doi: 10.1016/j. schres.2014.08.018

Lieslehto, J., Kiviniemi, V. J., Nordström, T., Barnett, J. H., Murray, G. K., Jones, P. B., et al. (2018). Polygenic risk score for schizophrenia and face-processing network in young adulthood. Schizophr. Bull. doi: 10.1093/schbul/sby139. [Epub ahead of print].

Martin, A. K., and Mowry, B. (2016). Increased rare duplication burden genomewide in patients with treatment-resistant schizophrenia. Psychol. Med. 46 (3), 469-476. doi: 10.1017/S0033291715001701

Need, A. C., Keefe, R. S., Ge, D., Grossman, I., Dickson, S., McEvoy, J. P., et al. (2009). Pharmacogenetics of antipsychotic response in the CATIE trial: a candidate gene analysis. Eur. J. Hum. Genet. 17 (7), 946-957. doi: 10.1038/ejhg.2008.264
Nucifora, F. C., Jr., Woznica, E., Lee, B. J., Cascella, N., and Sawa, A. (2018). Treatment resistant schizophrenia: clinical, biological, and therapeutic perspectives. Neurobiol. Dis. (18), 30494-30497. doi: 10.1016/j.nbd.2018.08.016 . [Epub ahead of print].

Ota, V. K., Spíndola, L. N., Gadelha, A., dos Santos Filho, A. F., Santoro, M. L., Christofolini, D. M., et al. (2012). DRD1 rs4532 polymorphism: a potential pharmacogenomic marker for treatment response to antipsychotic drugs. Schizophr Res. 142 (1-3), 206-208. doi: 10.1016/j.schres.2012.08.003

Pinheiro, D. S., Santos, R. D. S., de Brito, R. B., Cruz, A. H. D. S., Ghedini, P. C., and Reis, A. A. S. (2017). GSTM1/GSTT1 double-null genotype increases risk of treatment-resistant schizophrenia: a genetic association study in Brazilian patients. PLoS One 12 (8), e0183812. doi: 10.1371/journal.pone.0183812

Purcell, S. M., Moran, J. L., Fromer, M., Ruderfer, D., Solovieff, N., Roussos, P., et al. (2014). A polygenic burden of rare disruptive mutations in schizophrenia. Nature 506 (7487), 185-190. doi: 10.1038/nature12975

Radulescu, E., Jaffe, A. E., Straub, R. E., Chen, Q., Shin, J. H., Hyde, T. M., et al. (2018). Identification and prioritization of gene sets associated with schizophrenia risk by co-expression network analysis in human brain. Mol. Psychiatry. doi: 10.1038/s41380-018-0304-1. [Epub ahead of print].

Ranlund, S., Rosa, M. J., de Jong, S., Cole, J. H., Kyriakopoulos, M., Fu, C. H. Y., et al. (2018). Associations between polygenic risk scores for four psychiatric illnesses and brain structure using multivariate pattern recognition. Neuroimage. Clin. 20, 1026-1036. doi: 10.1016/j.nicl.2018.10.008

Remington, G., Lee, J., Agid, O., Takeuchi, H., Foussias, G., Hahn, M., et al. (2016). Clozapine's critical role in treatment resistant schizophrenia: ensuring both safety and use. Expert Opin. Drug Saf. 15 (9), 1193-1203. doi: 10.1080/14740338.2016.1191468

Richards, A., Horwood, J., Boden, J., Kennedy, M., Sellers, R., Riglin, L., et al. (2019). Associations between schizophrenia genetic risk, anxiety disorders and manic/ hypomanic episode in a longitudinal population cohort study. Br. J. Psychiatry 214 (2), 96-102. doi: 10.1192/bjp.2018.227

Ruderfer, D. M., Charney, A. W., Readhead, B., Kidd, B. A., Kähler, A. K., Kenny, P. J. et al. (2016). Polygenic overlap between schizophrenia risk and antipsychotic response: a genomic medicine approach. Lancet Psychiatry. 3 (4), 350-357. doi: 10.1016/S2215-0366(15)00553-2

Santoro, M. L., Ota, V., de Jong, S., Noto, C., Spindola, L. M., Talarico, F., et al. (2018). Polygenic risk score analyses of symptoms and treatment response in an antipsychotic-naive first episode of psychosis cohort. Transl. Psychiatry 8 (1), 174. doi: 10.1038/s41398-018-0230-7

Schizophrenia Psychiatric Genome-Wide Association Study (GWAS) Consortium (2011). Genome-wide association study identifies five new schizophrenia loci. Nat. Genet. 43 (10), 969-976. doi: 10.1038/ng.940

Schizophrenia Working Group of the Psychiatric Genomics Consortium (2014). Biological insights from 108 schizophrenia-associated genetic loci. Nature 511 (7510), 421-427. doi: 10.1038/nature13595

Taylor, M. J., Martin, J., Lu, Y., Brikell, I., Lundström, S., Larsson, H., et al. (2018). Association of genetic risk factors for psychiatric disorders and traits of these disorders in a Swedish population twin sample. JAMA Psychiatry. doi: 10.1001/ jamapsychiatry.2018.3652. [Epub ahead of print].

Teo, C., Zai, C., Borlido, C., Tomasetti, C., Strauss, J., Shinkai, T., et al. (2012). Analysis of treatment-resistant schizophrenia and 384 markers from candidate genes. Pharmacogenet. Genomics. 22 (11), 807-811. doi: 10.1097/FPC.0b013e3283586c04

Terzić, T., Kastelic, M., Dolžan, V., and Plesničar, B. K. (2015). Influence of 5-HT1A and 5-HTTLPR genetic variants on the schizophrenia symptoms and occurrence of treatment-resistant schizophrenia. Neuropsychiatr. Dis. Treat. 11, 453-459. doi: 10.2147/NDT.S76494

Velthorst, E., Froudist-Walsh, S., Stahl, E., Ruderfer, D., Ivanov, I., Buxbaum, J., et al. (2018). iPSYCH-Broad ASD Group, the IMAGEN consortium. Genetic risk for schizophrenia and autism, social impairment and developmental pathways to psychosis. Transl. Psychiatry 8 (1), 204. doi: 10.1038/s41398-018-0229-0

Vivian-Griffiths, T., Baker, E., Schmidt, K. M., Bracher-Smith, M., Walters, J., Artemiou, A., et al. (2019). Predictive modeling of schizophrenia from genomic data: comparison of polygenic risk score with kernel support vector machines approach. Am. J. Med. Genet. B Neuropsychiatr. Genet. 180 (1), 80-85. doi: 10.1002/ajmg.b.32705

Wang, S. H., Hsiao, P. C., Yeh, L. L., Liu, C. M., Liu, C. C., Hwang, T. J., et al. (2018). Polygenic risk for schizophrenia and neurocognitive performance in patients with schizophrenia. Genes Brain Behav. 17 (1), 49-55. doi: 10.1111/gbb.12401 
Wheeler, A., Humberstone, V., and Robinson, G. (2009). Outcomes for schizophrenia patients with clozapine treatment: how good does it get? J. Psychopharmacol. 23 (8), 957-955. doi: 10.1177/0269881108093588

Wimberley, T., Gasse, C., Meier, S. M., Agerbo, E., MacCabe, J. H., and Horsdal, H. T. (2017). Polygenic risk score for schizophrenia and treatment-resistant schizophrenia. Schizophr. Bull. 43 (5), 1064-1069. doi: 10.1093/schbul/ sbx007

Zhang, J. P., Lencz, T., Geisler, S., DeRosse, P., Bromet, E. J., and Malhotra, A. K. (2013). Genetic variation in BDNF is associated with antipsychotic treatment resistance in patients with schizophrenia. Schizophr Res. 146 (1-3), 285-288. doi: 10.1016/j.schres.2013.01.020

Zhang, J. P., Robinson, D., Yu, J., Gallego, J., Fleischhacker, W. W., Kahn, R. S., et al. (2018). Schizophrenia polygenic risk score as a predictor of antipsychotic efficacy in first-episode psychosis. Am. J. Psychiatry 176 (1), 21-28. doi: 10.1176/ appi.ajp.2018.17121363

Conflict of Interest Statement: The authors declare that the research was conducted in the absence of any commercial or financial relationships that could be construed as a potential conflict of interest.

Copyright (c) 2019 Pisanu and Squassina. This is an open-access article distributed under the terms of the Creative Commons Attribution License (CC BY). The use, distribution or reproduction in other forums is permitted, provided the original author(s) and the copyright owner(s) are credited and that the original publication in this journal is cited, in accordance with accepted academic practice. No use, distribution or reproduction is permitted which does not comply with these terms. 